\title{
CURRENT SINGULARITIES IN LINE-TIED THREE-DIMENSIONAL MAGNETIC FIELDS
}

\author{
I. J. D. CRAiG ${ }^{1}$ AND D. I. Pontin ${ }^{2}$ \\ ${ }^{1}$ Department of Mathematics, University of Waikato, Hamilton, New Zealand; i.craig@waikato.ac.nz \\ ${ }^{2}$ Division of Mathematics, University of Dundee, Dundee, UK; dpontin@maths.dundee.ac.uk \\ Received 2014 February 28; accepted 2014 May 8; published 2014 June 5
}

\begin{abstract}
This paper considers the current distributions that derive from finite amplitude perturbations of line-tied magnetic fields comprising hyperbolic field structures. The initial equilibrium on which we principally focus is a planar magnetic $X$-point threaded by a uniform axial field. This field is line-tied on all surfaces but subject to threedimensional (3D) disturbances that alter the initial topology. Results of ideal relaxation simulations are presented which illustrate how intense current structures form that can be related, through the influence of line-tying, to the quasi-separatrix layers (QSLs) of the initial configuration. It is demonstrated that the location within the QSL that attracts the current, and its scaling properties, are strongly dependent on the relative dimensions of the QSL with respect to the line-tied boundaries. These results are contrasted with the behavior of a line-tied 3D field containing an isolated null point. In this case, it is found that the dominant current always forms at the null, but that the collapse is inhibited when the null is closer to a line-tied boundary.
\end{abstract}

Key words: magnetic fields - magnetic reconnection - magnetohydrodynamics (MHD) - Sun: corona

Online-only material: color figure

\section{INTRODUCTION}

In a series of previous studies, the development of ideal current singularities in magnetic null points has been investigated using a mixture of analytic arguments and numerical experiments (Craig \& Litvinenko 2005; Pontin \& Craig 2005). It is well known that when magnetic equilibria of complex topology are perturbed, currents will be generated that can only be dissipated by magnetic reconnection (Priest \& Forbes 2000). Yet, in a highly conducting magnetic plasma such as the solar corona, reconnection can be effective only in highly localized regions of strong current density. In the case of three-dimensional (3D) magnetic null points, currents can form locally at the null in response to a magnetic collapse in the weak-field region, often forming quasi one-dimensional (1D) current sheets or quasicylindrical current tubes. The geometry of the current distributions derive from "spine" and "fan" structures that form the skeleton of the null-essentially the eigenstructure of the 3D field - and these different current structures define the forms the reconnection can take (e.g., Craig \& Fabling 1996; Pontin 2011). Accordingly, a useful route for understanding reconnection is to examine the near-singular current distributions that derive from perturbing 3D magnetic fields.

In a previous paper, Pontin \& Craig (2005) used an ideal Lagrangian magneto-frictional method to examine the singular current distributions that derive from perturbing two-dimensional (2D) and 3D compressible magnetic null points. One of the aims of that study was to quantify the extent to which finite gas pressure could inhibit the development of the current singularity, and thus presumably slow down the reconnection rate in the realistic case of a small but finite resistivity. Our present purpose is to complement this work by examining the stabilizing role of line-tying on the formation of current singularities. To do this, we initially consider perturbing a 2D planar $X$-point threaded by a uniform perpendicular field $B_{z}$. The equilibrium field has an ignorable $z$-coordinate, and contains no neutral point, but perturbations that alter the magnetic topology naturally lead to singular 3D current sheets. We consider two questions. (1) How effective is line-tying the axial field in determining the form and strength of the current singularity? (2) To what extent can these results be applied to line-tied fields containing 3D nulls?

In Section 2, we introduce the line-tied $X$-point geometry that forms the basis of our initial investigation. We point out that the presence of a line-tied, axial field component introduces quasi-separatrix layers (QSLs) that fundamentally alter the causal properties of the configuration. Relaxation simulations in 3D show that line-tying manifests itself by inhibiting the blow-up of the current density around the axis of the QSL. In Section 3, corresponding relaxation computations are performed for the case of a 3D null-point field. The relaxed current distribution now highlights a competition that develops between currents localized at the null and those localized at the line-tied footpoints. In this case, line-tying is effective in slowing the divergence of the "reconnective currents" that localize around the null. Our conclusions are presented in Section 4.

\section{2. $X$-POINT THREADED BY A UNIFORM AXIAL FIELD}

\subsection{The Equilibrium Configuration}

The simplest magnetic configuration of interest is the 2D potential $X$-point $\mathbf{B}=\nabla \psi \times \hat{\mathbf{z}}$ defined by the planar flux function $\psi=B_{0} x y$. We assume that the gas pressure is negligible but impose line-tying on the boundary of the region $-1 \leqslant x, y \leqslant 1$.

Consider adding a perturbation $\mathbf{b}=b_{0} x \hat{\mathbf{y}}$ to this field so that

$$
\psi(x, y)=B_{0} x y-b_{0} \frac{x^{2}}{2} .
$$

The separatrices-field lines threading the null that delineate regions of distinct flux - are no longer at right angles but are tilted through the additional angle

$$
\tan \theta=\frac{b_{0}}{2 B_{0}} .
$$

The equilibrium can be regained only by flux transfer across the separatrices. In a weakly resistive plasma, this involves an 
implosion of the disturbance toward the $X$-point, generating a current sheet at which magnetic reconnection occurs (McClymont \& Craig 1991; Hassam 1992). Note that if resistive effects are turned off, but some other form of damping is present, the final configuration comprises a singular distribution of current in the weak field regions close to the null (Green 1965; Syrovatskii 1971).

Now suppose that the initial equilibrium is threaded by a uniform axial field $B_{z}$ of infinite length:

$$
\mathbf{B}_{E}=\nabla \psi \times \hat{\mathbf{z}}+B_{z} \hat{\mathbf{z}}, \quad \psi=B_{0} x y .
$$

The null point is removed-fundamentally altering the causal properties of the configuration since the Alfvén speed is now non-vanishing — but a disturbance typified by (1) still alters the topology. That is, by projecting field lines onto a surface $z=$ constant, effective "separatrices" can be defined that delineate regions of distinct flux. Field lines on the effective separtrices extend to infinity in one direction rather than connecting back to the boundary as in the case of the planar $X$-point. The presence of the $B_{z}$ field introduces an extra pressure (of magnitude $B_{z}{ }^{2} / 2$ ) that resists the subsequent implosion. Detailed computations for small amplitude perturbations confirm that the magnetic pressure weakens the current singularity but cannot stop it from forming (McClymont \& Craig 1996; Craig \& Litvinenko 2005). This is not surprising given that the problem maintains the symmetry $\partial_{z}=0$ in the absence of line-tying. In this case, finite $B_{z}$ leads to an irrotational force $\nabla\left(B_{z}{ }^{2} / 2\right)$, acting much like gas pressure, that cannot balance the Lorentz force that drives the implosion. This argument fails only under highly restrictive conditions-for instance, 1D current layers-which are unlikely to apply in the present study.

The symmetry $\partial_{z}=0$ is broken when the axial field is linetied on upper and lower surfaces, $z= \pm z_{m}$, say. This leads to an equilibrium that has been studied for a number of years in the context of current sheet formation and 3D reconnection. More specifically, when contained within a bounded domain, the field (3) contains a new topological feature, the so-called QSL (Titov et al. 2002; Titov 2007). The QSL is centered on the $z$-axis and extends along the $y$-axis on the lower boundary and along the $x$-axis on the upper boundary (assuming $B_{0}, B_{z}>0$; Priest \& Démoulin 1995). More generally, QSLs can be related to geometrical squashing factors associated with field line mappings in line-tied magnetic configurations (Titov 2007). We note that in this fully 3D configuration there no longer exist regions of distinct magnetic flux - the boundaries between which correspond to discontinuities in the field line mapping - but the QSL delineates a thin layer across which this mapping has a strong variation.

Of interest to the present study is the current build-up due to driving motions on the field boundaries. Although QSLs provide natural surfaces for current accumulation in 3D line tied configurations such as Equation (3), there is no single point within the QSL that provides a focus for the current localization that derives from footpoint driving. Inverarity \& Titov (1997) demonstrated linearized solutions in which the field evolved through a sequence of force-free states in response to a slow driving flow on the $z$-boundaries. For some driving flows they found a current singularity of a logarithmic type. Of more direct relevance are the resistive MHD simulations of Galsgaard (2000), who subjected the lower $z$-boundary to various driving motions-including a simple shear flow that leads locally to a separatrix displacement as proposed in Equation (1). A strong current enhancement within the QSL was obtained.
Below we point out that the current structures obtained by Galsgaard (2000) bear a strong resemblance to the "relaxed" magnetic structures obtained in the present simulations. However, the form and intensity of the current structure obtained is likely to be strongly dependent on the form of perturbation applied to the system, as observed by Inverarity \& Titov (1997) and Galsgaard (2000). In what follows, we focus on what we call "topological disturbances," which are perturbations that disturb the location of the QSL (or in Section 3 the true separatrices).

\subsection{Magneto-frictional Relaxation}

Current structures are obtained by adding topological disturbances onto the line-tied equilibrium field (3) and following the subsequent evolution using an ideal Lagrangian scheme (Craig \& Sneyd 1986). The present version of the code uses fourth-order spatial differencing within a rectangular region $-1 \leqslant x, y \leqslant 1$ of height $2 z_{m}$ centered on the origin. Fluid particles on the boundaries are held fixed but those in the interior-driven by the $\mathbf{J} \times \mathbf{B}$ forces and subject to frictional damping - are followed until the computed Lorentz forces are negligible. The code features implicit time-stepping, satisfies $\nabla \cdot \mathbf{B}=0$ to machine accuracy, and is unconditionally stable. Effects due to finite gas pressures are assumed negligible.

The Lagrangian scheme works on the principle that fluid line elements evolve in the same way as $\mathbf{B} / \rho$ (where $\rho$ is the local mass density). Since fluid particle displacements $\xi(x, y, z)$ are the primary variables, we choose initial conditions according to the induction equation for small perturbations, namely,

$$
\mathbf{b}(x, y, z)=\nabla \times\left(\xi \times \mathbf{B}_{E}\right),
$$

where $\mathbf{B}_{E}$ is given by Equation (3). A displacement that reproduces Equation ( 1$)$ is provided by $\xi=b_{0} x /\left(2 B_{0}\right) \hat{\mathbf{y}}$. This form is conveniently extended into the computational $3 \mathrm{D}$ domain by assuming separable functions of $y$ and $z$ that vanish on the boundaries. It should be stressed that although perturbations constructed in this way are expected to provide a divergent current layer, the frictional relaxation could, at least potentially, evolve toward a force-free equilibrium that involves non-singular current structures.

We begin by considering the result of applying a perturbation of the form

$$
\xi(x, y, z)=\frac{b_{0}}{2 B_{0}} x\left(1-y^{2}\right)\left(1-\left(z / z_{m}\right)^{2}\right) \exp \left(-3 y^{2}\right) \hat{\mathbf{y}} .
$$

Although this displacement is even in $z$ this symmetry is not transferred to components of the current density or the Lorentz force.

\subsection{Relaxed Current Distributions}

The relaxation is performed using the parameters $B_{0}=1$ and $b_{0}=0.6$ for an axial field of strength $B_{z}=0.4$. These parameters, though comparable to those of Galsgaard (2000), are chosen mainly to highlight the role played by line-tying the axial field in determining the distribution of current in the relaxed configuration. Fields are taken to be "computationally relaxed" when the initial forces (of the order of unity) have declined by over three orders of magnitude.

Figure 1 illustrates the current structures obtained by taking $z_{m}=1$ using $N=81$ support points in each direction. Shown are isosurfaces of the current modulus at levels 1.3, 1.5, and 1.6, respectively. It is clear that the higher current densities are 

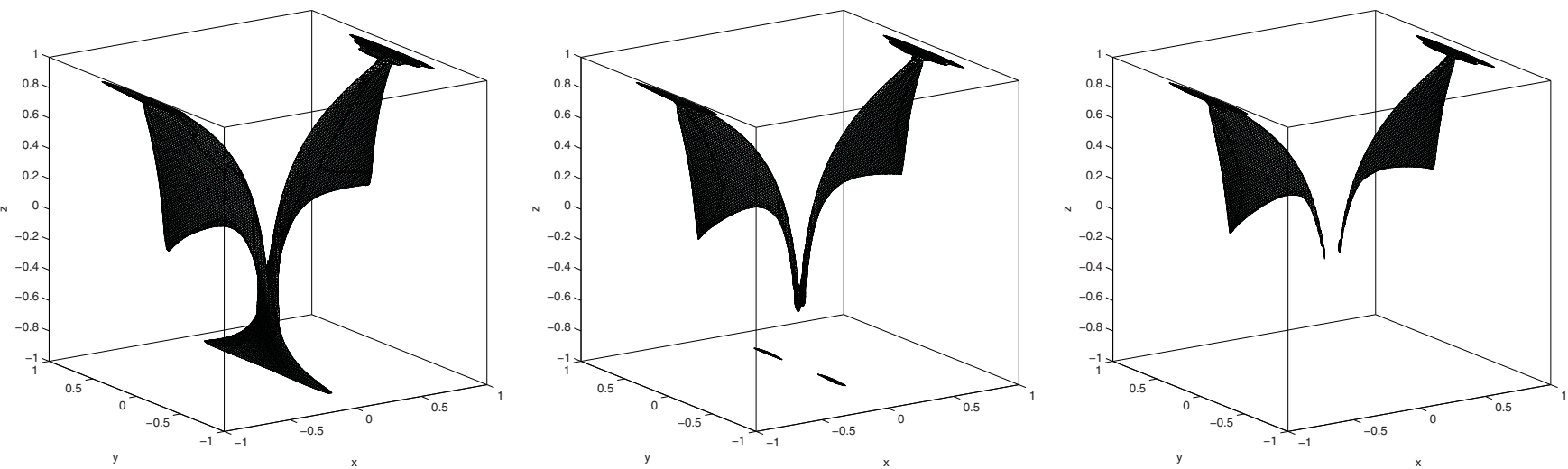

Figure 1. Surface current densities for half-length $z_{m}=1$. Shown from left to right are surfaces at levels 1.3, 1.5, and 1.6. It is clear that the highest concentrations of the current are limited to the upper reaches of the QSL Current accumulation along $z$-axis is relatively minor.
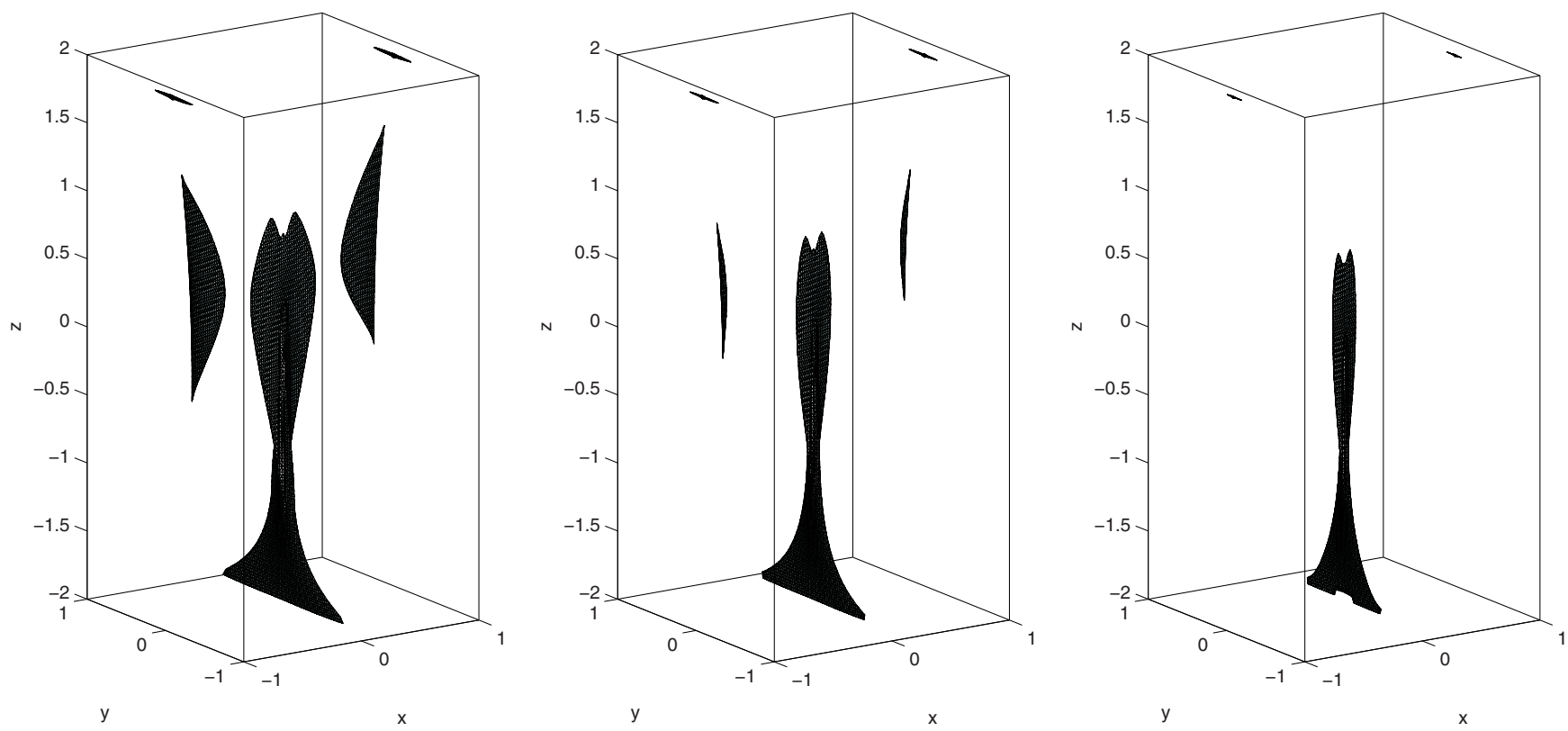

Figure 2. Surface current densities for half-length $z_{m}=2$. Shown from left to right are surfaces at levels 1.6, 1.8, and 2.0. In contrast to Figure 1, it is now the lower levels of the QSL that are delineated by the current distribution.

associated mainly with stresses in the vicinity of the upper linetied boundary $z=1$. In this example, the axial field effectively prevents the current localizing strongly toward the $z$-axis. Notably, these figures closely resemble Figure 3 of Galsgaard (2000), who uses the value $B_{z}=0.3$ over a fixed computational mesh (typically $-0.5 \leqslant x, y, z \leqslant 0.5$ ) without considering in detail the influence of the size of the domain.

Figure 2 illustrates the results of a computation in which $z_{m}=2$. The resolution of the previous figure is maintained-mesh points in the $z$-direction are increased in proportion to the extension in height - and all other parameters are left unchanged. The expectation is that the greater tube height should weaken the influence of axial line-tying. Current surfaces taken at $1.6,1.8$, and 2.0 confirm that, in contrast to Figure 1, current localization is now strongest along the tube axis. In this case, the current distribution delineates mainly the lower reaches of the QSL.

To interpret these results, recall that in the case of infinitely long tubes $\left(\partial_{z}=0\right)$, current accumulation at the planar null controls the reconnection rate in perturbed $X$-points. This rate $\eta J$ is known to be "fast"-invariant with resistivity $\eta$ - only if $B_{z}{ }^{2} / 2<\eta$ (McClymont \& Craig 1996). The presence of a finite computational domain, however, changes matters radically since, by line-tying the axial field, QSLs are introduced. It is these surfaces of steep gradient in the field line mapping that now provide sites for current accumulation. However, even in the present rather simple geometry, the location of the peak current within the QSL is affected by a combination of factors such as the length of the flux tube and the form of perturbation applied. Note also that if the resistivity were turned on, reconnection would involve field lines whose end points are anchored across different $z$-planes, as in the field line "flipping" of Priest \& Forbes (1992).

\subsection{Current Magnitude versus z-length}

We now examine more systematically how the maximum current densities on the mesh vary with changes in the height parameter $z_{m}$. Figure 3 shows how variations in $z_{m}$ define three distinct regimes. For $z_{m}>1.5$ there is a regime in which the current density, localized along the vertical axis of the QSL (as in Figure 2), shows a slow increase with increasing $z_{m}$. 


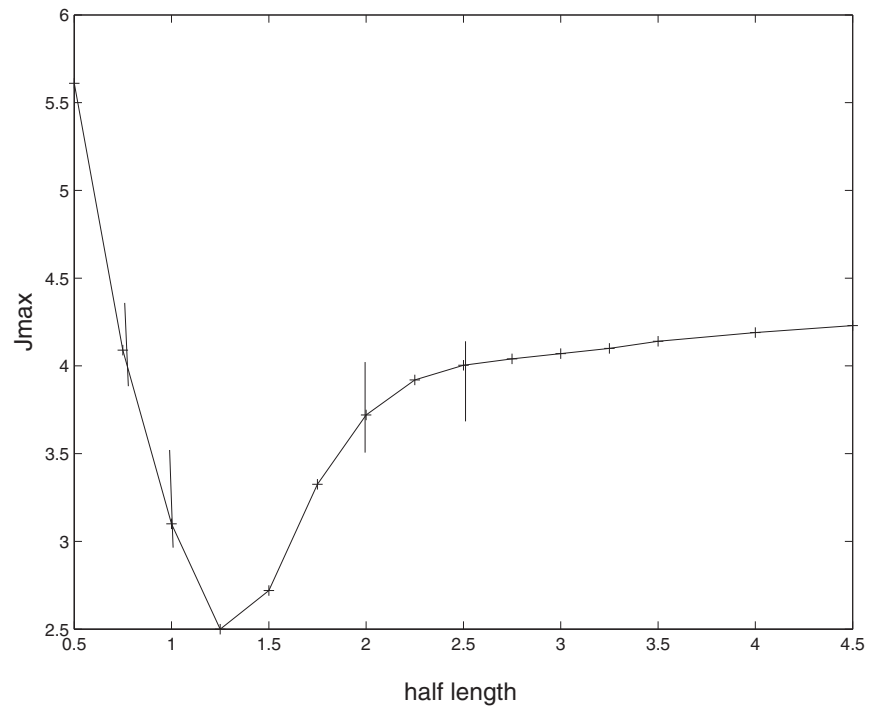

Figure 3. Surface current density vs. half-length $z_{m}$. For sufficiently long tubes $\left(z_{m}>1.5\right)$ the maximum current is located on the axis of the QSL and the peak current density slowly increases with tube length. For $z_{m}<1.5$ the footpoint currents associated with line-tying at the upper boundary become significant. These dominate for sufficiently short tubes and effectively prevent current localization on the tube axis.

For $z_{m}<1.2$ the current is concentrated toward the upper boundary footpoints (as in Figure 1) and decreases with increasing length. The implication is that, at sufficiently small lengths, the $B_{z}$-field is strong enough, and line-tying effective enough, to prevent the current localizing around the $z$-axis. In the intermediate regime $1.2<z_{m}<1.5$ there is a transition that reflects the competition between the interior and footpoint currents.

It should be stressed that the plots of Figure 3 are all computed for a fixed numerical resolution (so height increases are always achieved by increasing the mesh points in the $z$-direction). Independent of all other considerations, however, the absence of resistivity means that, in response to a topological disturbance such as Equation (5), the current densities in the relaxed configuration should diverge as the resolution increases (Craig \& Litvinenko 2005; Pontin \& Craig 2005). If the current structure in the relaxed field is truly singular then, to the extent that $B_{z}$ acts like an isotropic gas pressure, the current density is expected to approximate a power law $J \sim N^{\alpha}$ whose exponent weakens with the increasing strength of the axial field (Craig \& Litvinenko 2005). Line-tying $B_{z}$ on the upper and lower boundaries, however, introduces a magnetic tension force that is likely to slow this divergence. Of course, the divergent behavior could be quashed completely if, at sufficiently high resolution, the underlying current structure were to have a finite thickness. This occurs, for example, in a 1D current sheet halted by compressional effects due to gas pressure or finite $B_{z}$ (Craig \& Litvinenko 2005, Appendix A).

As already anticipated, current tends to accumulate at the location of the QSL in the field as identified by Priest \& Démoulin (1995). It is straightforward to show that for the equilibrium field $\left(b_{0}=0\right)$, the covariant squashing factor (between the planes $z= \pm z_{m}$ ) as defined by Titov (2007) takes the value

$$
Q_{\perp}(x=0, y=0)=2 \cosh \left(\frac{4 z_{m}}{B_{z}}\right)
$$

on the $z$-axis. Clearly, the squashing factor gets larger as $B_{z}$ is decreased or $z_{m}$ is increased. Therefore, in the regime $z_{m}>1.5$

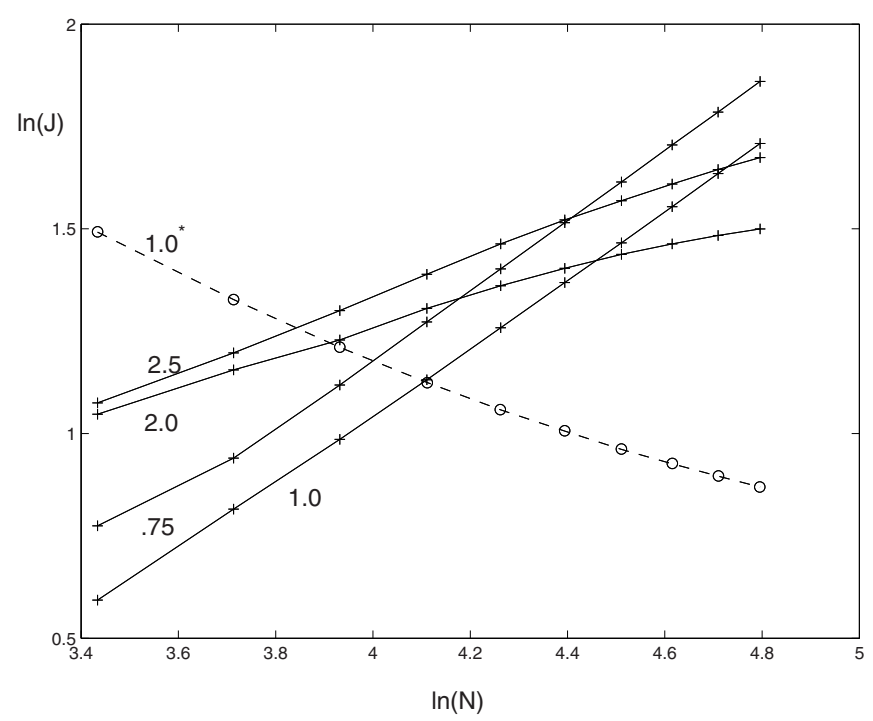

Figure 4. Divergence of the current density $J$ for various half-lengths $z_{m}$ as a function of the grid resolution $N$. Stronger divergences are obtained for (shorter) tubes which comprise localized footpoint currents. Shown for comparison is a convergence plot (dashed line labeled $z_{m}=1.0^{*}$ ) obtained using a nontopological disturbance for a tube of unit half-length (with $J$ multiplied by 50 for graphical purposes).

when the current accumulates in the center of the QSL, our results are consistent with the notion that the peak current in the QSL increases as the squashing factor increases. While this behavior is expected on intuitive grounds, it is far from clear that a direct link can be established between the squashing factor in the initial field and the current intensity that develops in response to the perturbation - see Démoulin (2006) for a discussion.

\subsection{Divergence of the Current Magnitude with Resolution}

Four vertical bars are marked on Figure 3. These indicate tube heights $z_{m}$ at which we have explored the divergence of the current density with resolution (as measured by $N$, the number of grid points across the tube axis). Figure 4 confirms divergences that approximate power law behavior for each of the four heights plotted. Slight roll-offs are present for the elongated tubes (say $z_{m} \geqslant 2$ ), but the shorter tubes-which resist current localization at the center of the tube-possess stronger, well defined divergences $\left(J \sim N^{0.83}\right.$ for $\left.z_{m}=0.75\right)$. This suggests that, independent of the tube length, by line-tying the $B_{z}$ field, we encourage current accumulation about the axial footpoints at the expense of current localization about the tube axis.

Finally, in Figure 4 we present a plot $\left(z_{m}=1.0^{*}\right)$ that shows the convergence obtained when the tube is subject to a nontopological disturbance. In this case, the original perturbation (5) has been modified to provide a "control" disturbance that guarantees vanishing displacements at the origin and at the tube footpoints. In this case, reconnection is not required to regain the initial equilibrium and we therefore expect no localized current enhancements to develop. Consistent with this, we see that in marked contrast to the divergence plots, the convergence of the current density (multiplied by fifty for graphical purposes) is accelerated by increases in resolution.

\subsection{The Form of the Perturbation}

It has already been noted that the current structures obtained by magnetic relaxation are likely to be sensitive to the form of the applied perturbation. Here we briefly consider two variations of the disturbance given by Equation (5). 


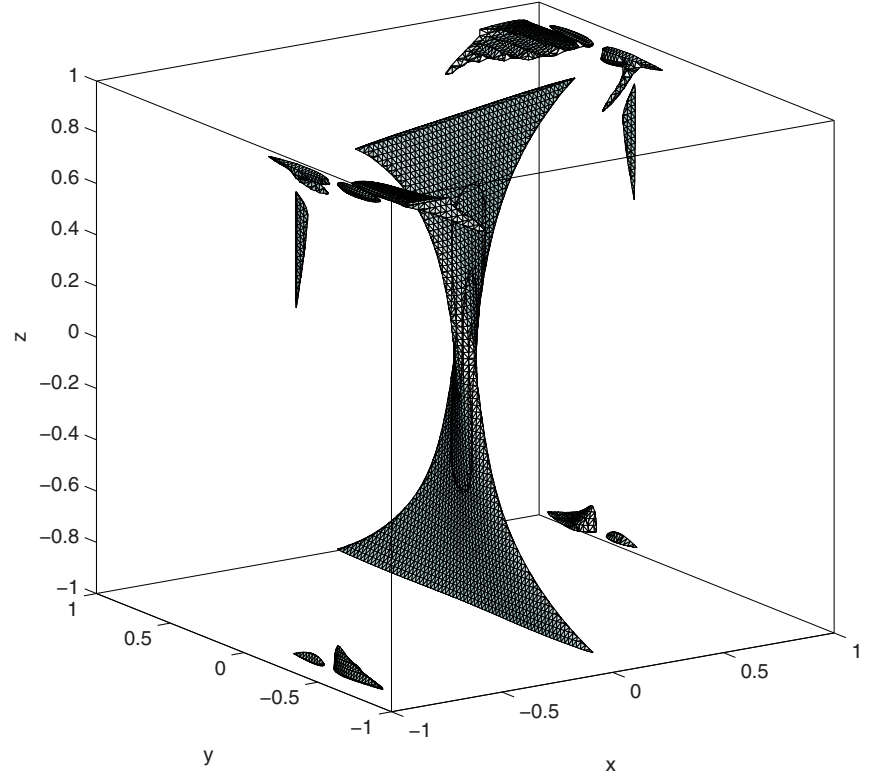

Figure 5. Isosurface of the current modulus at $36 \%$ of the maximum for a simulation in which the applied perturbation is non-zero on the $z$-boundaries-see Equation (7). Despite the well-represented QSL layer in the present figure $\left(z_{m}=1.0\right)$, we find that the QSL axis currents can dominate footpoint currents only when the tube is sufficiently long $\left(z_{m}>1.5\right)$.

Figure 5 shows the current structure obtained by turning off the $z$-dependence of the perturbation, specifically by taking

$$
\xi(x, y, z)=\frac{b_{0}}{2 B_{0}} x\left(1-y^{2}\right) \exp \left(-3 y^{2}\right) \hat{\mathbf{y}} .
$$

The entire QSL is well represented-compare Figure 1 for $z_{m}=1$-but localized currents attached to the footpoints are also clearly present. Although it is tempting to interpret these footpoint currents as numerical artifacts, it should be remembered that real magnetic stresses are present on the line-tied boundaries and that, according to the present computations, these stresses become increasingly dominant (compared with currents on the QSL axis) for the shorter tubes. Computations based on those in Section 2.3 again confirm that only when the effects of line-tying are weakened, specifically by taking $z_{m}>1.5$, is a dominant current distribution localized to the axis of the QSL realized.

Finally, in Figure 6, we show the result of adjusting the disturbance such that it is zero on all lateral boundaries. This would be equivalent in an MHD simulation to eliminating any driving from the lateral boundaries, but driving only from the $z$-boundaries. Specifically, we take

$$
\xi(x, y, z)=\frac{b_{0}}{2 B_{0}} x\left(1-y^{2}\right)\left(1-x^{2}\right) \exp \left(-3 x^{2}-3 y^{2}\right) \hat{\mathbf{y}} .
$$

In this case, the perturbation is non-zero only on the top and bottom surfaces (which, in previous studies of the QSL geometry, were often identified as fragments of the solar photosphere; Inverarity \& Titov 1997; Galsgaard 2000). The interesting aspect of the resultant current structure is that, in being concentrated to the upper regions of the tube, the distribution is rather insensitive to increases in the tube length (especially compared with Figure 3). That is, there appears to be a limit to the distance along the tube that the current can penetrate. The peak current diverges with resolution as before, but the scalings for tubes longer than $z_{m} \geqslant 1$ show only minimal variations. This is consistent with the results of Galsgaard (2000), who found little variation in the current structure obtained through a dynamical driving when the domain was doubled in length. On the other hand, for tubes with $z_{m} \ll 1$, the current appears to converge to a finite value, indicating that the underlying current layer is probably of finite thickness. This behavior clearly differs from our previous examples and may derive from the fact that the perturbation no longer involves displacements of the lateral footpoint boundaries. We return to a discussion of the possibility of non-singular QSL currents in Section 4 below.

\section{3D NULL POINTS}

\subsection{The Simulation Setup}

We now explore the current structures obtained by perturbing, nonlinearly, a fully 3D equilibrium field. Of particular interest is the extent to which the results of the line-tied $X$-point model of Section 2 can be applied to more general 3D magnetic nulls. An exact equivalence cannot be expected since, in the absence of a true magnetic null, all points in the QSL field considered above are causally related. We present only a preliminary study here, but point out that the competition between localized interior currents and the footpoint currents, so apparent in the previous analysis, is also a salient feature in the model outlined below. For the cases considered, however, the current at the null is always ultimately dominant.

Our initial condition is a potential magnetic field due to four flux patches on the lower boundary of the domain, $z=-z_{m}$. The field is generated by four point charges that lie outside the domain at $z<-z_{m}$. The resultant magnetic field is given by

$$
\mathbf{B}_{p}=\sum_{i=1}^{4} \epsilon_{i} \frac{\mathbf{x}-\mathbf{x}_{i}}{\left|\mathbf{x}-\mathbf{x}_{i}\right|^{3}}
$$

where $\mathbf{x}_{i}$ are the locations and $\epsilon_{i}$ are the strengths of the point charges. Here we take $\left\{\epsilon_{1}, \epsilon_{2}, \epsilon_{3}, \epsilon_{4}\right\}=\{-0.5,-0.5$, $1.05,-0.015\}$ and $\mathbf{x}_{1}=(0,0.6,-1.0), \mathbf{x}_{2}=(0,-0.6,-1.0)$, $\mathbf{x}_{3}=(0,0,-1.3), \mathbf{x}_{4}=(0,0,-0.7)$. In what follows, we therefore restrict the domain height parameter to $z_{m}<0.7$. The field structure is shown in Figure 7, and possesses a well defined spine and fan structure that emanates from the isolated null at $x=y=0, z=-0.18$.

The geometry of the magnetic field is significantly more complicated than the linear null considered in the previous study of Pontin \& Craig (2005), since the spine of the null curves down to intersect with the lower boundary. However, the advantage of this is that all field lines that pass close to the null are anchored at $z= \pm z_{m}$ at least at their spine end (and in many cases at both ends). As such, we can hold the domain dimensions in $x$ and $y$ fixed since the line-tying there is relatively passive in the formation of currents at the null, and make a more direct comparison with the results of Section 2. Regarding comparison with the study of Pontin \& Craig (2005), it is worth noting that the field in the fan plane of the null is relatively isotropic: the ratio of the fan eigenvalues is 0.84 .

We perturb our 3D equilibrium by adding the displacement

$\xi(x, y, z)=b_{0} x\left(1-y^{2}\right)\left(1-\left(z / z_{m}\right)^{2}\right) \exp \left(-3 y^{2}\right) \exp \left(-10 x^{2}\right) \hat{\mathbf{y}}$.

This perturbation has the effect of deforming the field in the vicinity of the null such that the spine and fan are no longer perpendicular. This leads to a Lorentz force that acts to increase 

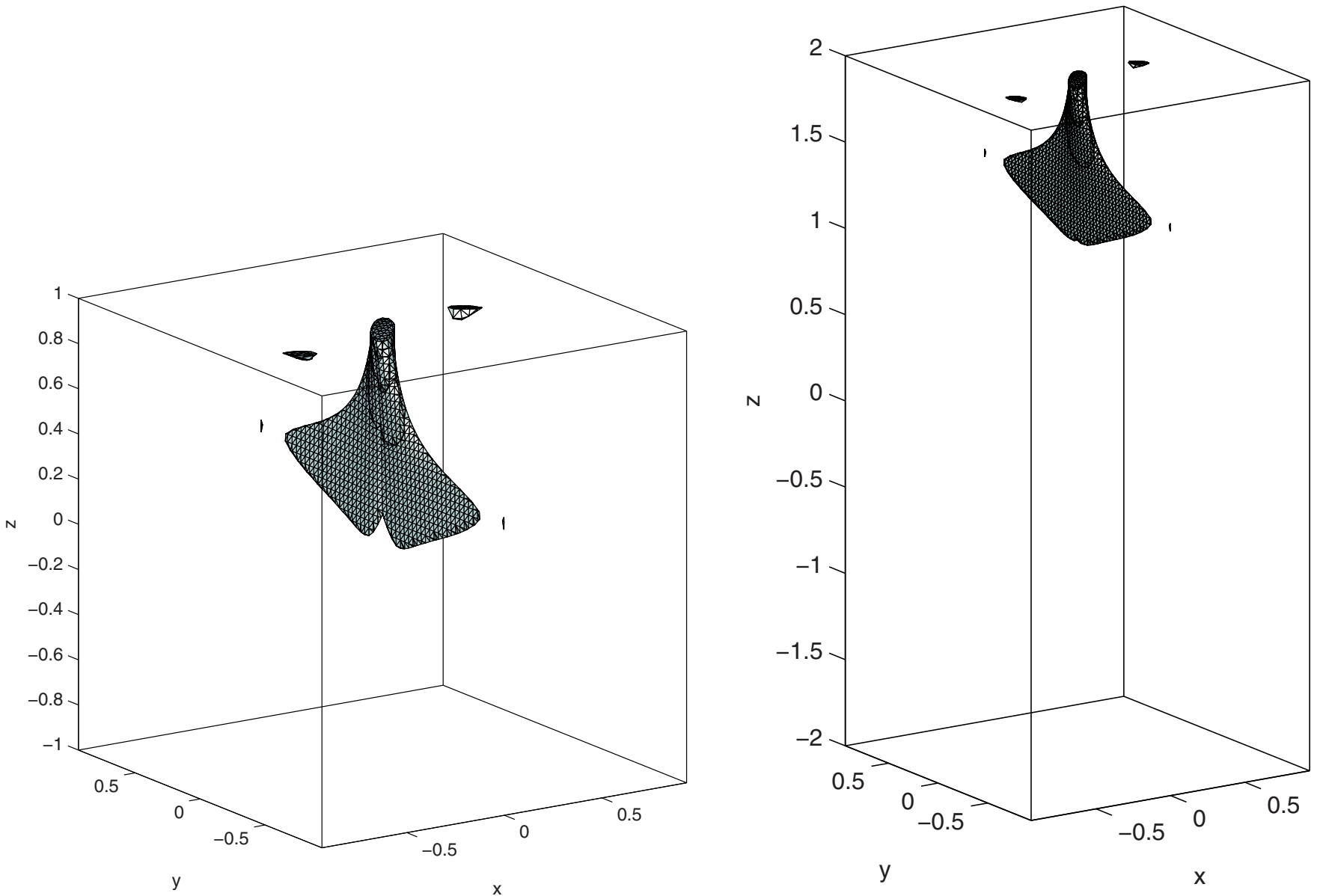

Figure 6. Isosurface of the current modulus at $70 \%$ of maximum for two simulations in which the applied perturbation is zero on the lateral boundaries but non-zero on the $z$-boundaries—see Equation (8)—for $z_{m}=1$ (left) and $z_{m}=2$ (right).

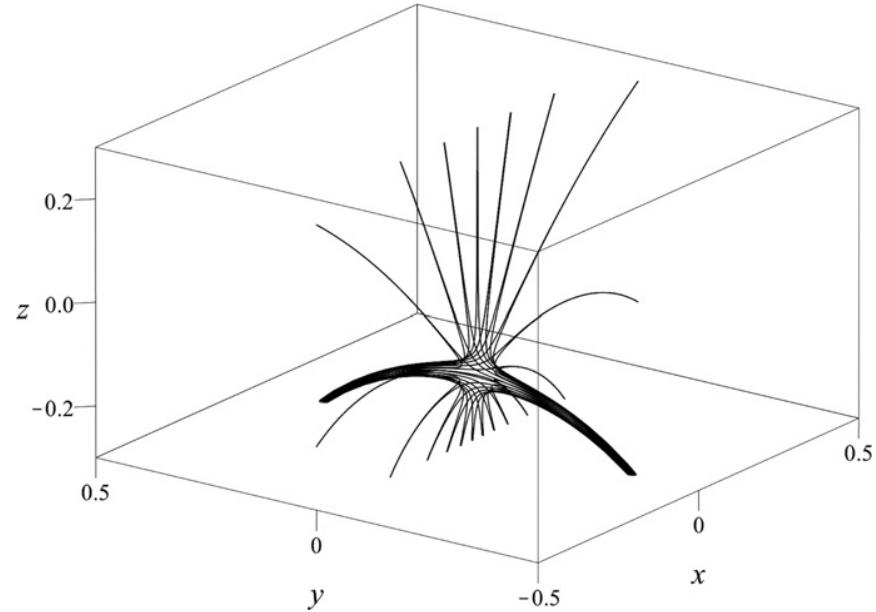

Figure 7. Spine-fan structure of the equilibrium magnetic field containing a 3D null point, with $z_{m}=0.3$.

the null collapse, as discussed in Pontin \& Craig (2005). The resulting non-equilibrium is then allowed to dynamically relax in the interior $\left(-1<x, y<1,|z|<z_{m}\right)$ while remaining linetied on all boundaries.

\subsection{Localization of the Current Density}

It is interesting that the $3 \mathrm{D}$ null field, when perturbed according to (10), has similar current localization properties to the planar null of Section 2. Notably, the current accumulates either at the null or toward the lower boundary in the vicinity of the footpoints of the spine and fan field lines. Which of these competing distributions is dominant can be expected to depend both on the height of the domain and the resolution of the computation, as well as the form of perturbation used.

Figure 8 shows a typical current localization for the case $z_{m}=0.5$. Note that in contrast to the discussion of Section 2, we now find it instructive to measure both the peak current at the null point and the peak current in the footpoint regions. Figure 9 compares the null and footpoint current distributions as a function of resolution in the case $z_{m}=0.5$. In common with the planar nulls of Section 2 (see, e.g., Figure 4), the current at the null eventually dominates, approximating a strong power law divergence $J \sim N^{1.64}$. In this case, the current at the spine and fan footpoints is strongest at low resolution $J \sim N^{0.58}$, but is readily overtaken as the resolution is increased.

We have also repeated the above computation for the case $z_{m}=0.3$. If we focus on providing a significant "reconnection current" at the null, then we would expect that current densities should weaken as the tube shortens (in the manner of regime 1, $z>1.5$, of Figure 3 ). However, what needs to be borne in mind is that lengthening the domain leads to larger field intensities, and therefore potentially larger currents, near the base of the domain. In this case, it therefore makes sense to consider not the absolute value of the current density but its scaling with resolution. For the simulations with $z_{m}=0.3$, line tying should now more strongly resist the collapse to singularity at the null, 

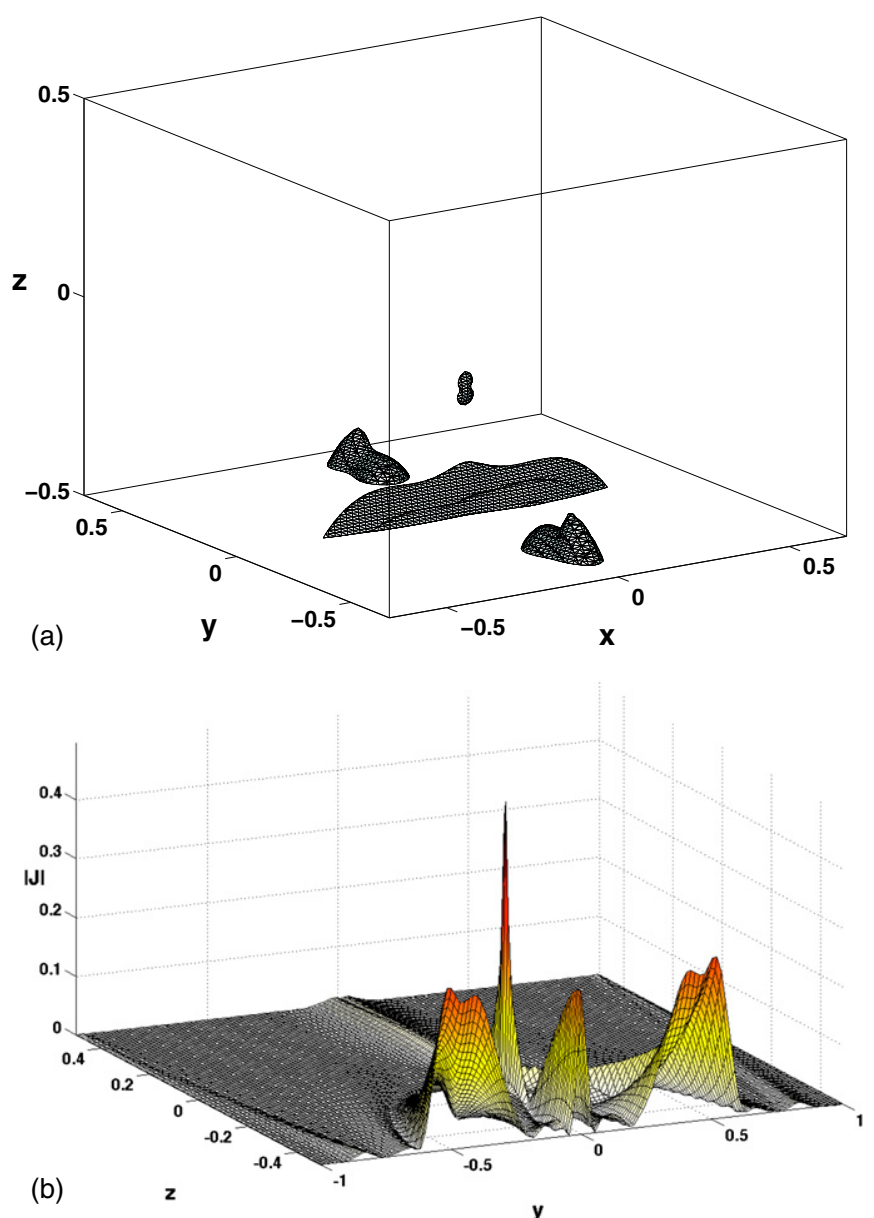

Figure 8. Current density $|\mathbf{J}|$ in the relaxed state of the field with the 3D null point. Mesh resolution is $121^{3}$ and $z_{m}=0.5$. (a) Isosurface of current at $25 \%$ of the maximum in the domain. (b) Surface plot of the current density in the mesh plane initially coincident with the $x=0$ plane.

(A color version of this figure is available in the online journal.)

and so we expect a weaker scaling of the peak current with resolution $N$. As compared to the curves for $z_{m}=0.5$, the blow up of the current density is indeed noticeably weaker, $J \sim N^{0.94}$, and the roll-over of the boundary current scaling even more pronounced. Importantly, it appears that for the null point field the reconnection current always eventually dominates, for sufficiently high resolution, in contrast (say) to the $z_{m}=.75$ and $z_{m}=1$ results for the linear $X$-point.

\section{DISCUSSION AND CONCLUSIONS}

We began by considering the action of a topological disturbance on a $2 \mathrm{D} X$-point threaded by a uniform axial field $B_{z}$. The initial field is line-tied on all boundary surfaces, and contains no magnetic null but rather a QSL aligned to the tube axis (here the $z$-axis). The absence of resistive effects means that very large currents can be expected to develop, in response to displacements of the lateral footpoints, over regions delineated by the QSL.

The literature appears divided over whether one should expect current densities that are formally singular within the QSL. Certainly, in the limit of an infinite domain length (corresponding to $Q_{\perp} \rightarrow \infty$, see Equation (6)), we expect a singular layer to develop (McClymont \& Craig 1996; Craig \& Litvinenko 2005). Notably, working in a finite-length domain, a logarithmic current singularity was obtained by Inverarity \&

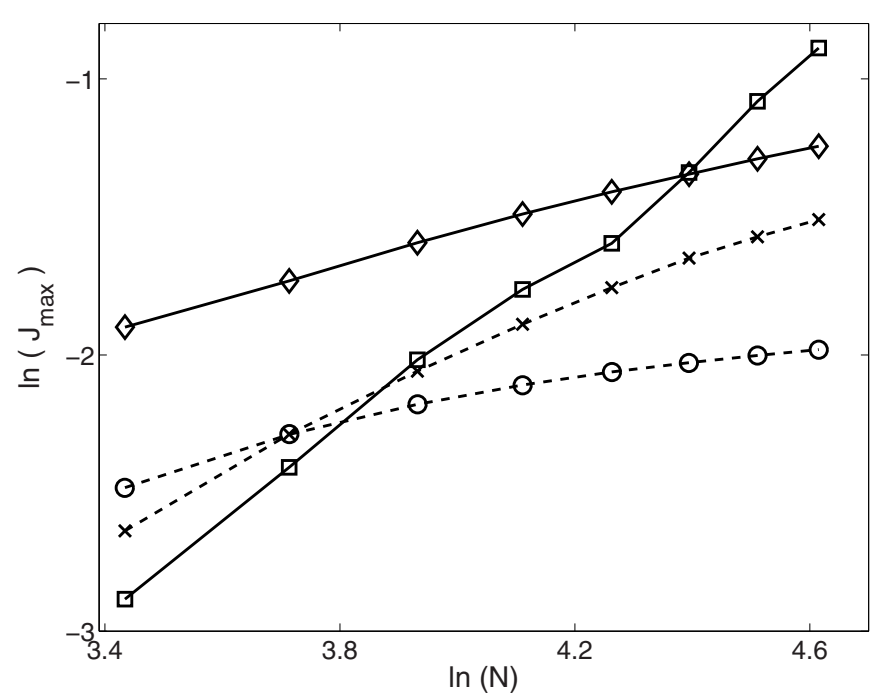

Figure 9. Scaling of the peak current density $J$ for $z_{m}=0.5$ (solid lines) and $z_{m}=0.3$ (dashed lines). Plotted are the current at the null (squares for $z_{m}=0.5$, crosses for $z_{m}=0.3$ ), and in the boundary current concentrations (diamonds for $z_{m}=0.5$, circles for $z_{m}=0.3$ ).

Titov (1997) for a particular form of boundary driving. Other studies, in contrast, propose that, in response to a dynamic driving from the boundaries, the current in the QSL should form an intense but finite layer whose thickness is in some way related to the thickness of the QSL (e.g., Titov et al. 2003; Galsgaard et al. 2003; Aulanier et al. 2005). This proposal was recently examined by Effenberger et al. (2011), who used an AMR code to follow the current collapse in a hyperbolic flux tube (defined by the intersection of two QSLs). Even at very high levels of grid refinement, no saturation of the current layer was observed. Indeed, while intuitively one expects the intensity of the current in the layer that forms in the QSL to be proportional to the squashing factor $Q$, a concrete theoretical demonstration remains elusive, as discussed by Démoulin (2006). What is more, the particular location within the QSL that most favorably attracts the current has not received much attention (though see Effenberger et al 2011) except in a handful of highly symmetric geometries.

In the ideal relaxation simulations described in Section 2, we find that for axial fields $B_{z}$ of comparable strength to the planar field, magneto-frictional relaxation provides two distinct outcomes for the relaxed current distribution, depending on the length $2 z_{m}$ of the tube and the form of the perturbation. If the tube is short enough $\left(z_{m}<1\right.$, for the examples studied), current localization on the tube axis is inhibited, and currents become attached mainly to the line-tied footpoints on the upper and lower surfaces. This behavior is not likely to be favorable to the onset of strong magnetic reconnection.

Current accumulation on the axis of the QSL can occur, however, despite the influence of line-tying, provided the tube is sufficiently long. For $z_{m}>2$ currents on the tube axis can completely detach from the upper and lower boundary surfaces. Longer tubes now lead to larger axial currents and potentially faster reconnection. Although the relaxed current distribution becomes near-singular, the smallness of the plasma resistivity in coronal plasmas means that frictional collapse should provide a useful guide to the strength and morphology of 3D reconnective currents. This view is supported by the transient resistive computations of Galsgaard (2000) which 
provide current structures consistent with QSLs that closely resemble the present results.

Whether the current sheet obtained after an ideal relaxation is formally singular is difficult to determine from a numerical simulation. A current layer of finite width would be expected to show a "roll-over" in the peak current divergence with resolution-that is, for sufficiently high resolution the peak current should converge yielding a force-free solution comprising sharp but non-singular current layers. What can be said is that for the limited resolution available to us, and for all simulations in which the lateral boundaries are perturbed, we have observed no sign of any such roll-over. Only in the case of short tubes (with low $Q$ ), perturbed only on the $z$-boundaries, do we observe current convergence indicating an underlying layer of finite width.

In Section 3, we explored line-tying in the context of a perturbed $3 \mathrm{D}$ magnetic null. In this case, the null point, as opposed to the QSL of Section 2, is able to provide a unique focus for the magnetic stresses. Relaxation computations confirm that current accumulates not only at the null but also in the footpoint regions associated with the line-tied boundaries. Null-point currents, however, are found to be the most intense. Indeed, a collapse to a singular current layer at the null is indicated in all simulations, despite the inhibiting influence of line-tying which acts to weaken the divergence of the peak current with resolution. Accordingly, when the effects of linetying are weakened-essentially by increasing in the height of the null point above the line-tied boundary-the reconnective currents at the null are significantly enhanced. Given the strong interest in factors affecting the speed of the magnetic reconnection, it will be of some interest to check the veracity of these findings in more general, weakly resistive, 3D plasmas.

Comments by Yuri Litvinenko have been much appreciated. D.P. acknowledges the financial support of a Philip Leverhulme Prize.

\section{REFERENCES}

Aulanier, G., Pariat, E., \& Démoulin, P. 2005, A\&A, 444, 961

Craig, I. J. D., \& Fabling, R. B. 1996, ApJ, 462, 969

Craig, I. J. D., \& Litvinenko, Y. E. 2005, PhPl, 12, 032301

Craig, I. J. D., \& Sneyd, A. D. 1986, ApJ, 311, 451

Démoulin, P. 2006, AdSpR, 37, 1269

Effenberger, F., Thust, K., Arnold, L., Grauer, R., \& Dreher, J. 2011, PhPl, 18,032902

Galsgaard, K. 2000, JGR, 105, 5119

Galsgaard, K., Titov, V. S., \& Neukirch, T. 2003, ApJ, 595, 506

Green, R. M. 1965, in IAU Symp. 22, Stellar and Solar Magnetic Fields, ed. R. Lust (Amsterdam: North-Holland), 398

Hassam, A. B. 1992, ApJ, 199, 159

Inverarity, G. W., \& Titov, V. S. 1997, JGR, 102, 22285

McClymont, A. N. M., \& Craig, I. J. D. 1991, ApJL, 371, L41

McClymont, A. N. M., \& Craig, I. J. D. 1996, ApJ, 466, 487

Pontin, D. I. 2011, AdSpR, 47, 1508

Pontin, D. I., \& Craig, I. J. D. 2005, PhPl, 12, 072112

Priest, E. R., \& Démoulin, P. 1995, JGR, 100, 23443

Priest, E. R., \& Forbes, T. G. 1992, JGR, 97, 1521

Priest, E. R., \& Forbes, T. G. 2000, Magnetic Reconnection (Cambridge: Cambridge Univ. Press)

Syrovatskii, S. I. 1971, Sov. Phys. JETP, 33, 933

Titov, V. S. 2007, ApJ, 660, 863

Titov, V. S., Galsgaard, K., \& Neukirch, T. 2003, ApJ, 582, 1172

Titov, V. S., Hornig, G., \& Démoulin, P. 2002, JGR, 107, 1164 